\title{
Universiteit
}

Leiden

The Netherlands

\section{Competition between MPS1 and microtubules at kinetochores regulates spindle checkpoint signaling}

Hiruma, Y.; Sacristan, C.; Pachis, S.T.; Adamopoulos, A.; Kuijt, T.; Ubbink, M.; ... ; Kops, G.J.P.L.

\section{Citation}

Hiruma, Y., Sacristan, C., Pachis, S. T., Adamopoulos, A., Kuijt, T., Ubbink, M., ... Kops, G. J. P. L. (2015). Competition between MPS1 and microtubules at kinetochores regulates spindle checkpoint signaling. Science, 348(6240), 1264-1267. doi:10.1126/science.aaa4055

Version: $\quad$ Publisher's Version

License: $\quad$ Licensed under Article 25fa Copyright Act/Law (Amendment Taverne)

Downloaded from: $\quad$ https://hdl.handle.net/1887/3197405

Note: To cite this publication please use the final published version (if applicable). 
25. A. V. Zaytsev, L. J. Sundin, K. F. DeLuca, E. L. Grishchuk, J. G. DeLuca, J. Cell Biol. 206, 45-59 (2014).

26. S. A. Kawashima, Y. Yamagishi, T. Honda, K. Ishiguro, Y. Watanabe, Science 327, 172-177 (2010)

27. Y. Yamagishi, T. Honda, Y. Tanno, Y. Watanabe, Science 330 , 239-243 (2010)

28. R. Karess, Trends Cell Biol. 15, 386-392 (2005).

29. S. Kemmler et al., EMBO J. 28, 1099-1110 (2009).

\section{ACKNOWLEDGMENTS}

We thank C. Brautigam for assistance with isothermal titration calorimetry and microscale thermophoresis, H. Ball for peptide synthesis, and the Animal Resource Center on campus for antibody production. H.Y. is an investigator with the Howard Hughes Medical Institute. This work is supported by the Cancer Prevention and Research Institute of Texas (RP110465-P3 and RP120717-P2) and the Welch Foundation (I-1441)

\section{SUPPLEMENTARY MATERIALS}

www.sciencemag.org/content/348/6240/1260/suppl/DC1

Materials and Methods

Figs. S1 to S8

References (30-35)

1 December 2014; accepted 7 May 2015

10.1126/science.aaa4029

\section{Competition between MPS1 and microtubules at kinetochores regulates spindle checkpoint signaling}

\author{
Yoshitaka Hiruma, ${ }^{1,2,3 *}$ Carlos Sacristan, ${ }^{2,3 *}$ Spyridon T. Pachis, ${ }^{2,3}$ \\ Athanassios Adamopoulos, ${ }^{1}$ Timo Kuijt, ${ }^{2,3}$ Marcellus Ubbink, ${ }^{4}$ Eleonore von Castelmur, ${ }^{1}$ \\ Anastassis Perrakis, ${ }^{1} \dagger$ Geert J. P. L. Kops $^{2,3} \dagger$
}

\section{Cell division progresses to anaphase only after all chromosomes are connected to spindle} microtubules through kinetochores and the spindle assembly checkpoint (SAC) is satisfied. We show that the amino-terminal localization module of the SAC protein kinase MPS1 (monopolar spindle 1) directly interacts with the HEC1 (highly expressed in cancer 1) calponin homology domain in the NDC80 (nuclear division cycle 80) kinetochore complex in vitro, in a phosphorylationdependent manner. Microtubule polymers disrupted this interaction. In cells, MPS1 binding to kinetochores or to ectopic NDC80 complexes was prevented by end-on microtubule attachment, independent of known kinetochore protein-removal mechanisms. Competition for kinetochore binding between SAC proteins and microtubules provides a direct and perhaps evolutionarily conserved way to detect a properly organized spindle ready for cell division.

A ttachment of microtubules to kinetochores of meiotic and mitotic chromosomes is essential for segregating a single copy of the genetic material to each of the daughter cells during cell division. This process is surveyed by the spindle assembly checkpoint (SAC), which orchestrates the assembly of an anaphase inhibitor $(1,2)$ that delays mitotic progression until all kinetochores are attached to microtubules $(3,4)$. Stable interaction of microtubules to all kinetochores silences the SAC, allowing anaphase to proceed. A key unresolved question is how the SAC machinery distinguishes attached from unattached kinetochores. In metazoa, poleward transport of SAC proteins by the dynein motor complex may contribute to extinguishing kinetochore-SAC signaling when microtubules have attached (5). However, kinetochore dynein is not widely conserved $(1,6)$, and SAC protein removal and silencing can occur without kinetochore dynein in human cells $(7,8)$. Kinetochore phosphatases are required to silence the

Division of Biochemistry, Netherlands Cancer Institute, 1066 CX Amsterdam, Netherlands. ${ }^{2}$ Molecular Cancer Research, University Medical Center Utrecht, 3584 CG Utrecht, Netherlands. ${ }^{3}$ Cancer Genomics Netherlands, University Medical Center Utrecht, 3584 CG Utrecht, Netherlands. ${ }^{4}$ Leiden Institute of Chemistry, Leiden University, Post Office Box 9502, 2300 RA Leiden, Netherlands.

*These authors contributed equally to this work. †Corresponding author. E-mail: g.j.p.I.kops@umcutrecht.nl (G.J.P.L.K.); a.perrakis@nki.nl (A.P.)
SAC in fungi and metazoa (9-11), but there is no attachment.

The SAC relies on the kinetochore-localized protein kinase MPS1 (monopolar spindle 1). Failure to remove it from kinetochores prevents SAC silencing and timely anaphase onset $(12,13)$. The microtubule-binding NDC80 (nuclear division cycle 80) complex [NDC80-C, which consists of HEC1 (highly expressed in cancer 1), NUF2 (nuclear filament-related 2), and SPC24 and SPC25 (spindle pole body component 24 and 25)] is needed for MPS1 localization to the kinetochores (14-18). The N-terminal calponin homology (CH) domain of human HECl is essential for that interaction (14), as also in Saccharomyces cerevisiae $(19,20)$. The MPS1 tetratricopeptide repeat (TPR) domain and a 62-amino acid N-terminal extension (NTE) are required for localizing MPS1 to kinetochores (14).

To test whether the NTE-TPR localization module of MPS1 binds directly to the NDC80-C, we expressed ${ }^{15} \mathrm{~N}$-labeled MPS1 N-terminal domain variants-namely MPS1 ${ }^{\text {TPR }}$, MPS1 $^{\text {NTE-TPR }}$, and MPS1 ${ }^{\text {TPR-CTE }}$ (CTE, C-terminal extension) (Fig. 1A and fig. S1A) (for details on all constructs, see the supplementary materials and methods). The corresponding ${ }^{1} \mathrm{H}^{-15} \mathrm{~N}$ heteronuclear single quantum coherence spectra showed dispersed peaks, as expected for the TPR structure (Fig. 1, B and $\mathrm{C}$ ). The ${ }^{1} \mathrm{H}^{15}{ }^{15}$ spectra recorded in the presence of the "Broccoli" variant of the NDC80-C evidence that they are regulated by microtubule
[NDC80-C $\left.\mathrm{C}^{\text {Broccoli }}(21)\right]$ showed peak broadening, leading to the disappearance of many peaks (Fig. 1, $\mathrm{D}$ and $\mathrm{E}$, and fig. S1B), which is indicative of the formation of a complex with higher molecular mass. Peak changes were also observed for binding to the "Bonsai" complex [NDC80 $\left.{ }^{\text {Bonsai }}(22)\right]$ (fig. S1, C to E).

We determined the strength of the interaction of the localization module of MPS1 and NDC80-C by microscale thermophoresis (MST) (all affinities determined in this study are summarized in table S1). The same variants as those used for the nuclear magnetic resonance (NMR) experiments (Fig. 1 F and fig. S2, A and B) and MPS1 ${ }^{\text {NTE-TPR-CTE }}$ (Fig. 1G) showed weak binding affinities (3 to $11 \mu \mathrm{M}$ ) when titrated to fluorescently labeled $\mathrm{NDC} 80^{\text {Broccoli. }}$. Relatively higher affinity was observed for NTE-containing constructs, consistent with our previous demonstration of NTE importance for MPS1 function (14) and with NMR spectra that show more changes in the presence of the NTE (Fig. 1E). Titrating NDC $80^{\text {Broccoli }}$ to fluorescently labeled full-length MPS1 showed similar affinity to that of the TPR-containing constructs $(\sim 10 \mu \mathrm{M})$ (fig. S2C). However, when NDC80 ${ }^{\text {Broccoli }}$ was titrated to fluorescently labeled $\mathrm{MPSI}^{\text {NTE-TPR-CTE }}$, we did not observe clear binding (Fig. 2A).

The N-terminal localization module of MPS1 is heavily phosphorylated, and cyclin-dependent kinase 1, Aurora B, Polo-like kinase 1 (PLK1), and MPS1 itself have been implicated in regulating localization of MPS1 to kinetochores $(14,15,20,22,23)$. Because most potential phosphorylation sites within NTE conform to an MPS1 or PLK1 consensus sequence, we used recombinant MPS1 to phosphorylate the N-terminal MPS1 localization module. Whereas titrating NDC80-C ${ }^{\text {Broccoli }}$ to labeled unphosphorylated MPS1 ${ }^{\text {NTE-TPR-CTE }}$ had resulted in no binding (Fig. 2A), exposing NDC80$\mathrm{C}^{\text {Broccoli }}$ to phosphorylated, labeled MPS1 ${ }^{\text {NTE-TPR-CTE }}$ (pMPS1 $^{\text {NTE-TPR-CTE }}$ ) resulted in clear interaction with an affinity of $\sim 150 \mathrm{nM}$ (Fig. 2A). The affinity of NDC80-C $\mathrm{C}^{\text {Broccoli }}$ to $\mathrm{pMPST}^{\mathrm{TPR}-\mathrm{CTE}}$ remained undetectable, indicating that the phosphorylation events leading to increased affinity are in the NTE. Phosphorylated full-length MPS1 had similar affinity $(\sim 210 \mathrm{nM})$ as pMPS1 ${ }^{\text {NTE-TPR-CTE }}$ for NDC80-C $\mathrm{C}^{\text {Broccoli }}$ (fig. S2D). In addition, the interaction of pMPS1 ${ }^{\text {NTE-TPR-CTE }}$ with NDC80-C ${ }^{\text {Broccoli }}$ was outcompeted by excess amounts of unlabeled $\mathrm{MPS1}^{\text {NTE-TPR-CTE }}$ (fig. S3A), indicating that the unphosphorylated and phosphorylated forms at least partially share the interaction site. Finally, pMPS1 ${ }^{\text {NTE-TPR-CTE }}$ also interacted with NDC80$\mathrm{C}^{\text {Bonsai }}$ (fig. S3B), as well as with only the $\mathrm{CH}$ domain of HEC1 (Fig. 2B). Collectively, these results 
show that phosphorylation of the NTE of MPS1 increases affinity toward NDC80-C by at least a factor of 20 and that a main MPS1 interaction site in the outer kinetochore exists in the $\mathrm{HECl} \mathrm{CH}$ domain.

We next tested whether microtubules could directly prevent the interaction of MPS1 to HEC1, which is known to contact microtubules (22). Binding of either MPS1 ${ }^{\text {NTE-TPR-CTE }}$ or pMPS1 ${ }^{\text {NTE-TPR-CTE }}$ to $\mathrm{NDC} 80^{\text {Broccoli }}$ was compromised by the presence of taxol-stabilized microtubules, in a manner dependent on microtubule concentration and polymerization time (Fig. $2 \mathrm{C}$ and fig. $\mathrm{S} 3, \mathrm{C}$ and D).

To test whether competition between MPS1 and microtubules for NDC80-C binding also occurs in cells, we examined whether MPS1 and microtubules could bind kinetochores simultane- ously. MPS1 amounts at kinetochores in prometaphase cells were high on unattached and laterally attached kinetochores but were almost undetectable on kinetochores that displayed clear end-on attachments (Fig. 3, A and B). Expression of the $\mathrm{Phe}^{258} \rightarrow \mathrm{Ala}^{258}$ mutant of Spindly, which specifically prevents activity of the kinetochore-localized pool of dynein (7), did not prevent microtubule
A
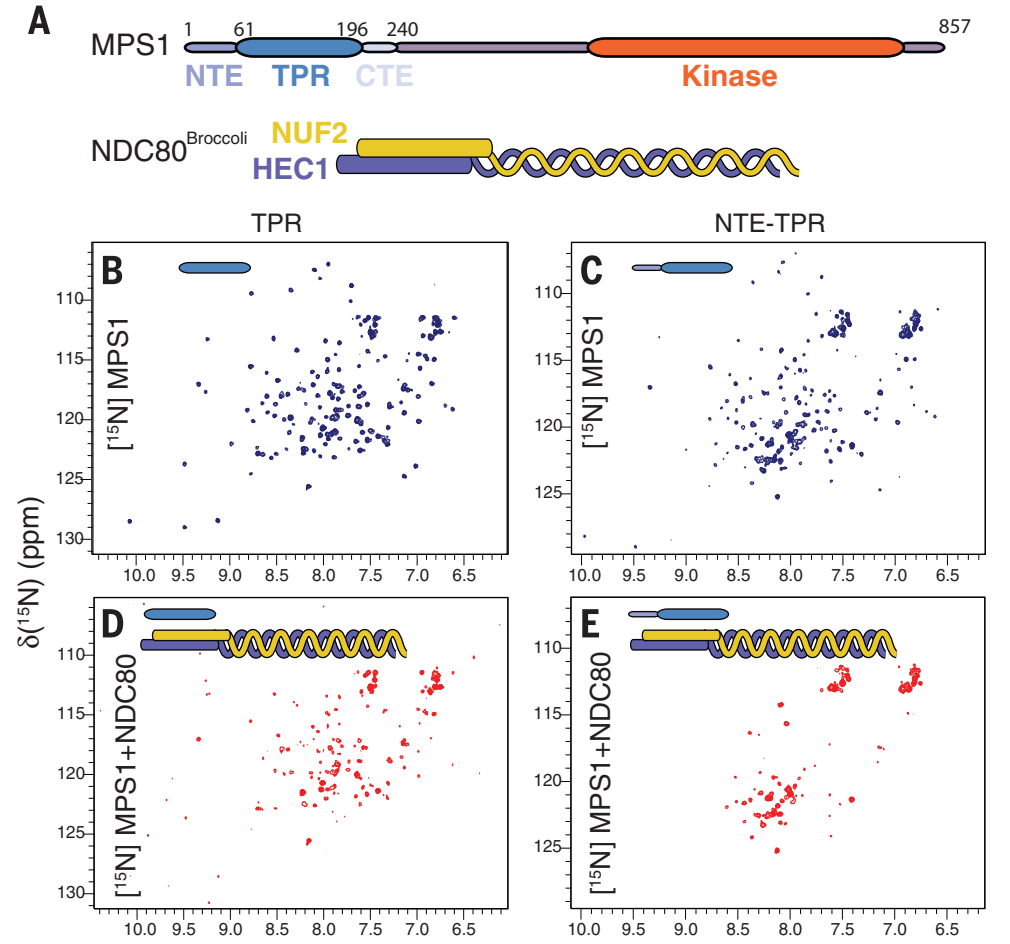

$\delta\left({ }^{1} \mathrm{H}\right)(\mathrm{ppm})$
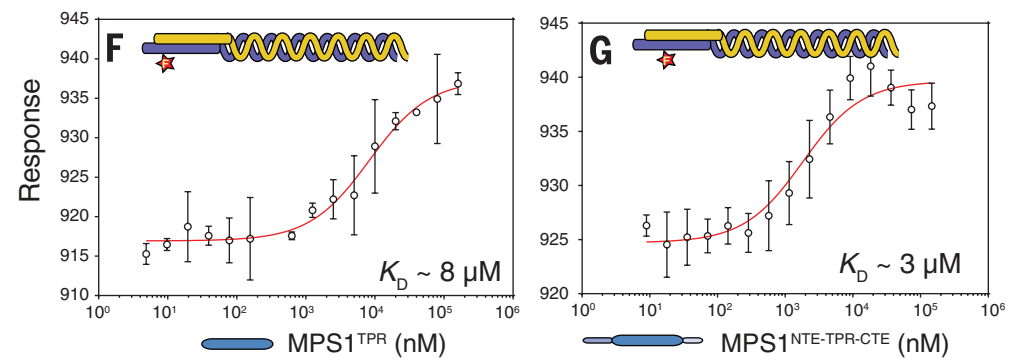

Fig. 1. Interaction of the $\mathrm{N}$-terminal localization module of MPS1 with the NDC80 complex. (A) Diagrams of MPS1 and NDC80-C ${ }^{\text {Broccoli. }}$ (B to E) NMR spectra of $\left[{ }^{15} \mathrm{~N}\right]$ MPS1 variants, alone or mixed with NDC80-C Broccoli. A $\sim 10 \%$ molar excess of NDC80-C was used for the complex measurements. $\delta$, chemical shift; ppm, parts per million. (F and $\mathbf{G}$ ) MST binding curves of MPS1 variants titrated against fluorescent $\mathrm{NDC} 80-\mathrm{C}^{\text {Broccoli }}$. Error bars show SDs from a triplicate experiment. $K_{\mathrm{D}}$, dissociation constant.
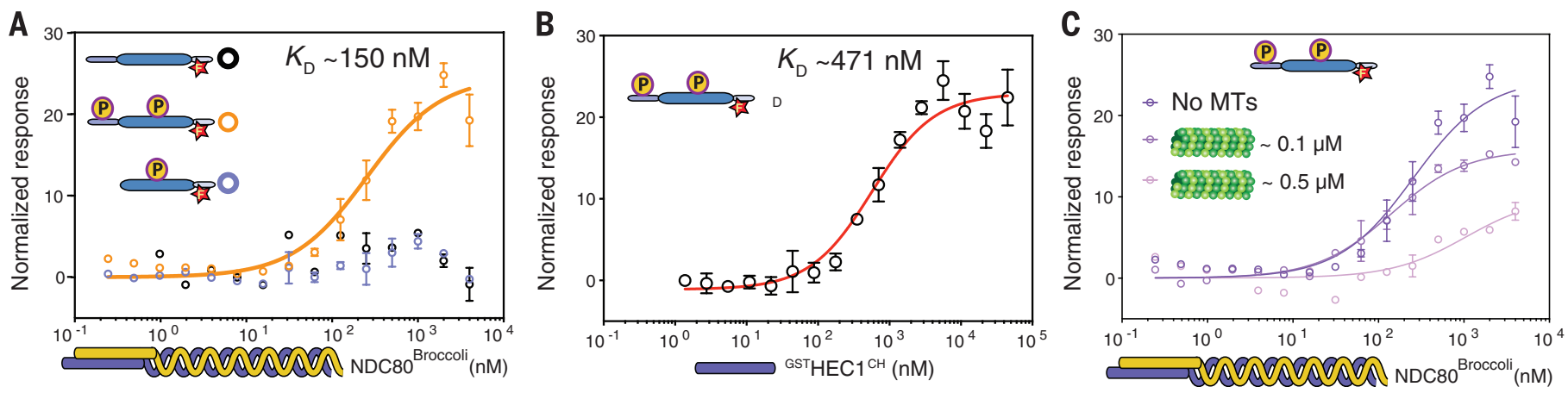

Fig. 2. Diminished binding of phosphorylated MPS1 to the NDC80 complex by addition of microtubules. (A) MST binding curves for NDC80-C Broccoli titrated against the indicated fluorescent MPS1 variants. (B) MST binding curves for the glutathione S-transferase (GST)-HEC1 CH domain titrated against

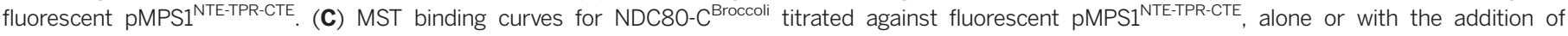
microtubules. Error bars show SDs from a triplicate experiment, except in (C) (duplicate experiment). MTs, microtubules; P, phosphate. 
A

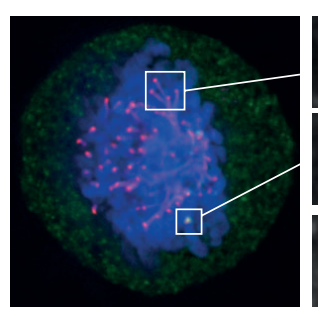

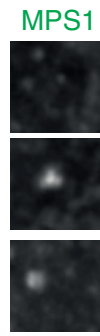
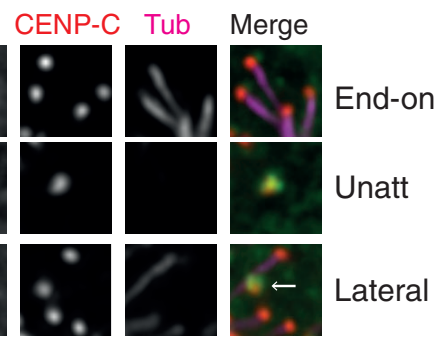

Merge
B

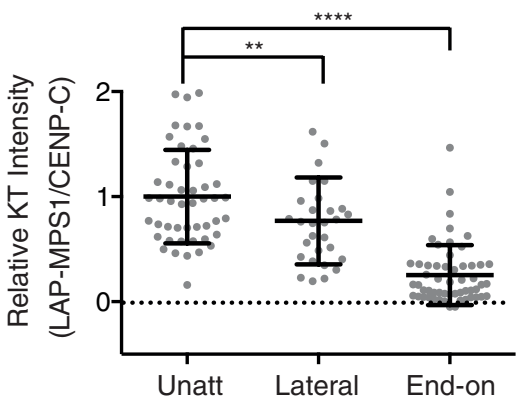

C
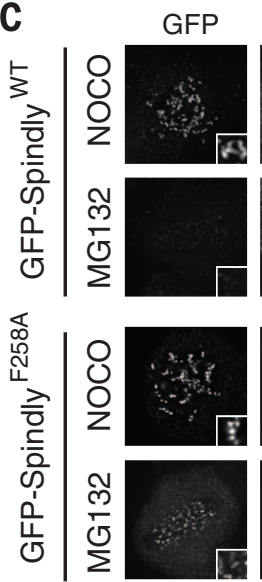
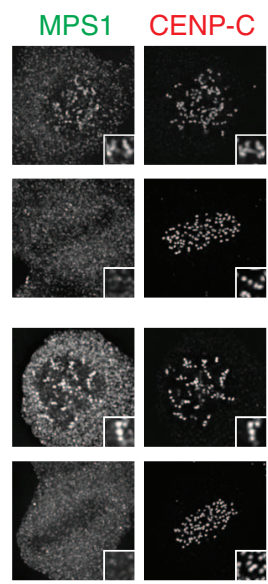
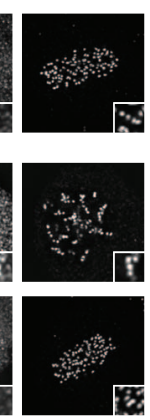
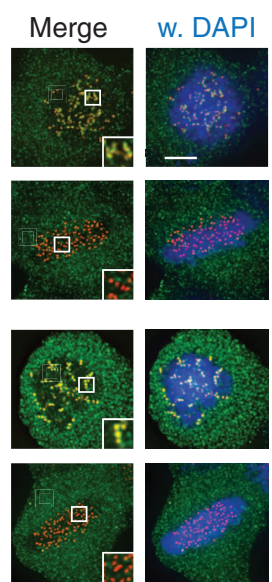

D

Spindly WT F258A WT F258A WT F258A WT F258A

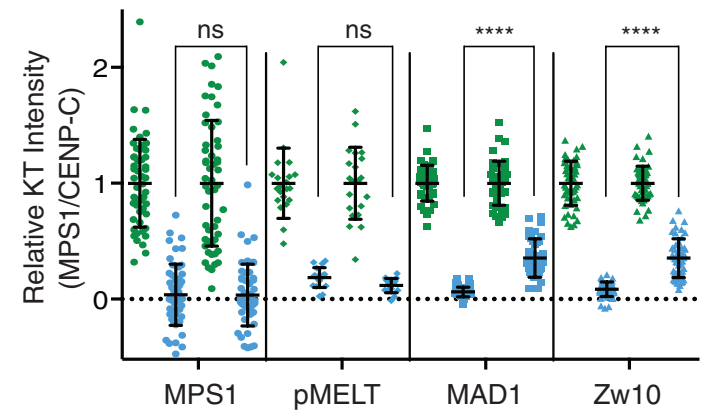

G
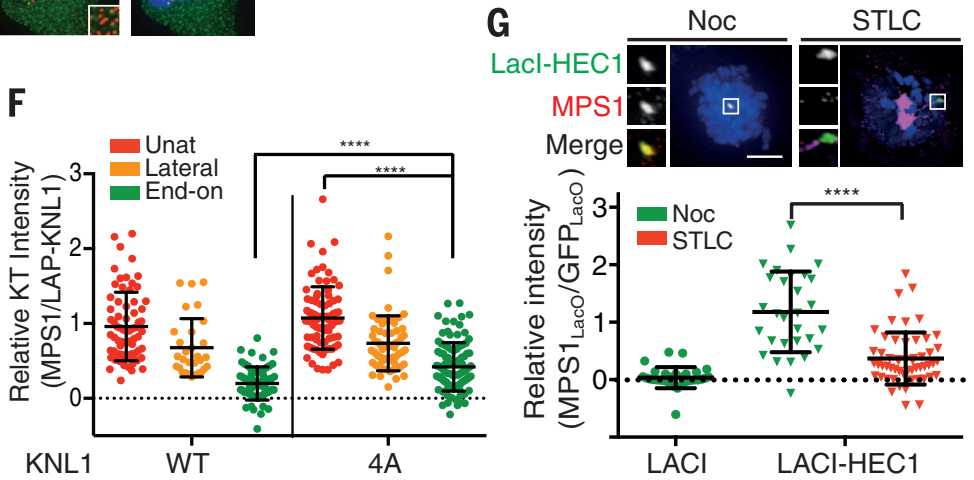

Fig. 3. Microtubule attachment-dependent delocalization of MPS1 from kinetochores, independent of dynein and phosphatase activity. (A to F) Representative images [(A), (C), and (E)] and quantification $( \pm S D)[(B),(D)$, and $(F)$ ] of protein immunolocalization in HeLa cells subjected to cold treatment $[(A)$ and $(B)]$, HeLa FLP-In cells expressing Spindly variants and treated with nocodazole [NOCO; green in (D)] or MG132 [blue in (D)] for 1 hour $[(C)$ and $(D)]$, or HeLa FLP-In cells expressing KNL1 variants [(E) and (F)]. The arrow in (A) indicates a laterally attached kinetochore. Tub, tubulin; GFP, green

fluorescent protein; WT, wild type; DAPI, 4',6-diamidino-2-phenylindole; F258A, $\mathrm{Phe}^{258} \rightarrow \mathrm{Ala}^{258}$; 4A, KNL1-4A; L; lateral, U; unattached, E; end-on attached. (G) Representative images and quantification $( \pm S D)$ of protein localization in U2OS-LacO cells expressing Lacl-LAP-HEC1 and treated with nocodazole or STLC. Cells expressing comparable levels of the Lacl proteins were selected for analysis. Asterisks indicate significance (analysis of variance with Tukey's multiple comparison test). ${ }^{*} P<0.01$; ${ }^{*}{ }^{*} P<0.0001$; ns, not significant. Scale bars in (C), (E), and (G), $5 \mu \mathrm{m}$.

attachment from removing MPS1 from kinetochores (Fig. 3, C and D), whereas it reduced the release of MAD1 (mitotic arrest deficient 1) and ZW10 (zeste white 10) (Fig. 3D and fig. S4A). Similar results were obtained by depletion of the dynactin subunits Arp1 or $\mathrm{p} 150^{\text {Glued }}(8,14)$ or by treatment of cells with the small-molecule dynein inhibitor ciliobrevin D (24) (fig. S4, B to E). Ciliobrevin D caused high frequency of kinetochores bound to the microtubule lattice, and these kinetochores had substantial amounts of MPS1 (fig. S4D). This verified that initial lateral attachments, which in animal cells do not involve the NDC80-C, did not dislodge MPS1 from kinetochores.

Displacement of MPS1 from attached kinetochores coincided with dephosphorylation of one of its key SAC substrates, KNL1 (kinetochore null 1) (Fig. 3D and fig. S4A). Precluding localization of the main SAC-silencing phosphatase PP1 (protein phosphatase 1) to kinetochores by expressing KNL1-4A (10), however, did not prevent microtubules from inhibiting MPS1 kinetochore binding (Fig. 3, E and F).

Decorating ectopic LacO arrays on the arm of chromosome 1 with LacI-tethered HEC1 was sufficient to localize endogenous MPS1 to those arrays in the absence of microtubules (Fig. 3G and fig. S4F) (14). Allowing microtubules from a monopolar spindle to engage in interactions with the LacI-HEC1 molecules on the LacO arrays resulted in delocalization of MPS1 (Fig. 3G). Notably, the $\mathrm{LacO}$ arrays were devoid of dynein (fig. S4F). These data support the hypothesis that MPS1 and microtubules compete for binding to NDC80-C in cells.

To identify which residues in HEC1 bind MPS1, we reasoned that because of the preferential binding of phosphorylated MPS1, hydrogen donors within HEC1 were likely candidates. Based on the structure of NDC80-C bound to microtubules (19), we designed four $\mathrm{NDC} 80^{\text {Broccoli }}$ mutants carrying clustered substitutions in the $\mathrm{HEC1} \mathrm{CH}$ domain, either directly in the interface with tubulin (M1) or peripheral to the interface (M2 to M4) (Fig. 4, A and B). Whereas M1 and $\mathrm{M} 3$ displayed a similar affinity for $\mathrm{PMPS1}{ }^{\text {NTE-TPR-CTE }}$ as wild-type $\mathrm{NDC} 80^{\mathrm{Broccoli}}, \mathrm{M} 2$ and, in particular, M4 had reduced affinities (Fig. 4C and fig. S5A). M4 was compromised in recruiting endogenous 

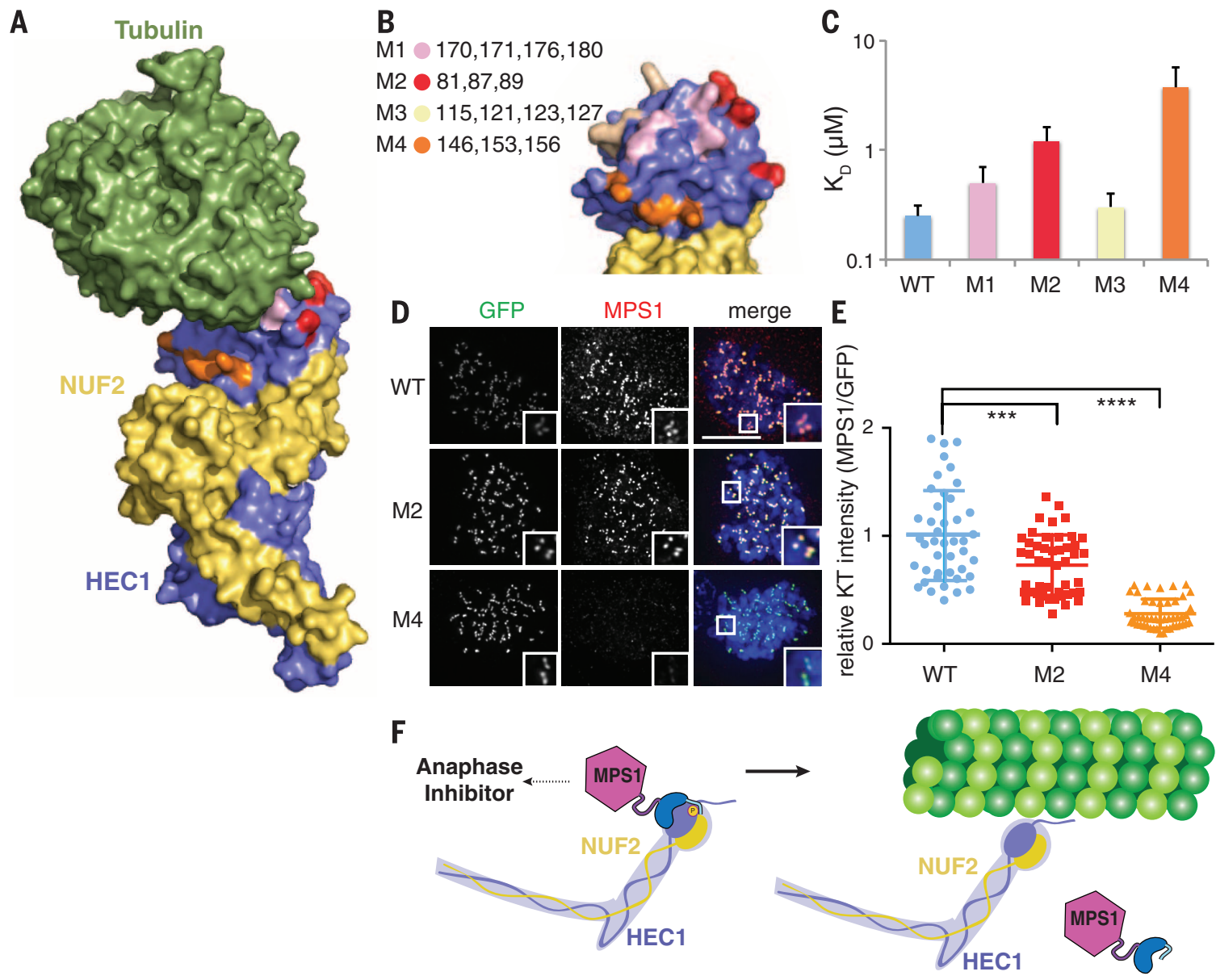

Fig. 4. Mutations in the $\mathbf{C H}$ domain of HEC1 preclude MPS1 binding. (A and B) Surface view of the NDC80 complex bound to tubulin (Protein Data Bank identification number: 3IZO) (A) or the HEC1 region close to tubulin (B), with the four HEC1 cluster mutants (M1 to M4) annotated in color. (C) Relative affinity of

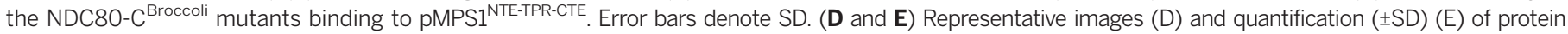
localization in HeLa Flp-IN cells expressing the indicated HEC1 variants and treated with nocodazole. ${ }^{* *} P<0.001$; ${ }^{* * *} P<0.0001$. (F) Model for how microtubules inhibit signaling by the SAC kinase MPS1.

MPS1 to LacO arrays (fig. S5, B and C) and endogenous kinetochores (Fig. 4D) and was unable to support a robust SAC response (fig. S5D). However, M4 remained capable of binding microtubules in vitro (fig. S5E) and supporting stable kinetochoremicrotubule interactions in cells (fig. S5F). These findings show that the HEC1-MPS1 interface is in close proximity to but is not identical to the HEC1microtubule interface (fig. S5G) and suggest that inhibition of MPS1-HEC1 interaction by microtubules is at least partly noncompetitive in nature.

Our data reveal a mechanism for how kinetochore-microtubule interactions inhibit production of the anaphase inhibitor. MPS1 directly binds the outer kinetochore NDC80-C in a phosphorylation-dependent manner, orchestrating SAC signaling by ensuring localization of SAC proteins and by promoting assembly and stability of the anaphase inhibitor (25). As MPS1 cycles dynamically on and off the kinetochore (12), endon attachments of microtubules to the NDC80-C can directly prevent rebinding of MPS1 (Fig. 4F). To extinguish the SAC, delocalization of MPS1 likely needs to coincide with removal of key downstream SAC effectors from kinetochores and with dephosphorylation of its substrates and perhaps of MPS1 $(10,26)$.

\section{REFERENCES AND NOTES}

1. M. Vleugel, E. Hoogendoorn, B. Snel, G. J. P. L. Kops, Dev. Cell 23, 239-250 (2012).

2. N. London, S. Biggins, Nat. Rev. Mol. Cell Biol. 15, 736-747 (2014).

3. E. A. Foley, T. M. Kapoor, Nat. Rev. Mol. Cell Biol. 14, 25-37 (2013)

4. C. Sacristan, G. J. P. L. Kops, Trends Cell Biol. 25, 21-28 (2015)

5. B. J. Howell et al., J. Cell Biol. 155, 1159-1172 (2001).

6. J. A. Raaijmakers, R. H. Medema, Chromosoma 123, 407-422 (2014).

7. R. Gassmann et al., Genes Dev. 24, 957-971 (2010).

8. J. A. Raaijmakers, M. E. Tanenbaum, R. H. Medema, J. Cell Biol. 201, 201-215 (2013).

9. J. S. Rosenberg, F. R. Cross, H. Funabiki, Curr. Biol. 21, 942-947 (2011)

10. W. Nijenhuis, G. Vallardi, A. Teixeira, G. J. P. L. Kops, A. T. Saurin, Nat. Cell Biol. 16, 1257-1264 (2014).

11. A. Espert et al., J. Cell Biol. 206, 833-842 (2014).

12. N. Jelluma, T. B. Dansen, T. Sliedrecht, N. P. Kwiatkowski, G. J. P. L. Kops, J. Cell Biol. 191, 281-290 (2010).

13. D. Ito, Y. Saito, T. Matsumoto, Proc. Natl. Acad. Sci. U.S.A. 109 , 209-214 (2012)

14. W. Nijenhuis et al., J. Cell Biol. 201, 217-231 (2013).

15. A. T. Saurin, M. S. van der Waal, R. H. Medema, S. M. A. Lens, G. J. P. L. Kops, Nat. Commun. 2, 316-319 (2011).

16. S. Martin-Lluesma, V. M. Stucke, E. A. Nigg, Science 297, 2267-2270 (2002)
17. S. Santaguida, C. Vernieri, F. Villa, A. Ciliberto, A. Musacchio, EMBO J. 30, 1508-1519 (2011)

18. T. Zhu et al., J. Biol. Chem. 288, 36149-36159 (2013).

19. G. M. Alushin et al., Nature 467, 805-810 (2010).

20. S. Kemmler et al., EMBO J. 28, 1099-1110 (2009).

21. J. C. Schmidt et al., Dev. Cell 23, 968-980 (2012).

22. C. Ciferri et al., Cell 133, 427-439 (2008).

23. Q. Xu et al., Mol. Biol. Cell 20, 10-20 (2009).

24. A. J. Firestone et al., Nature 484, 125-129 (2012).

25. W. Lan, D. W. Cleveland, J. Cell Biol. 190, 21-24 (2010).

26. M. Maldonado, T. M. Kapoor, Nat. Cell Biol. 13, 475-482 (2011).

\section{ACKNOWLEDGMENTS}

We thank I. Cheeseman, R. Gassmann, S. Lens, R. Medema, and A. Musacchio for reagents; the Kops, Perrakis, Lens, and Biggins labs for discussions; S. Biggins for support; and H. Yu for sharing unpublished data. The work was supported by grants ERC-StG KINSIGN, NWO-Vici 865.12.004, KWF 2012-5427. SNF PBBSP3133408, and NWO-Vici 700.58.441.

\section{SUPPLEMENTARY MATERIALS}

www.sciencemag.org/content/348/6240/1264/suppl/DC1 Materials and Methods

Figs. S1 to S5

Table S1

Reference (27)

2 December 2014; accepted 7 May 2015

10.1126/science.aaa4055 


\title{
Science
}

\section{Competition between MPS1 and microtubules at kinetochores regulates spindle checkpoint signaling}

Yoshitaka Hiruma, Carlos Sacristan, Spyridon T. Pachis, Athanassios Adamopoulos, Timo Kuijt, Marcellus Ubbink, Eleonore von Castelmur, Anastassis Perrakis and Geert J. P. L. Kops

Science 348 (6240), 1264-1267.

DOI: $10.1126 /$ science.aaa4055

\begin{abstract}
How cells sense connected chromosomes
Cells have a "checkpoint" that pauses cell division until all chromosomes are properly arranged on the mitotic spindle to allow precise distribution of one copy of each chromosome to each daughter cell. Hiruma et al. and Ji et al. explain the molecular mechanism by which cells sense that they are ready to divide. The protein kinase MPS1 associates with a protein complex at the kinetochore of the chromosome. Its activity produces signals that pause the cell cycle. When the chromosome becomes properly attached to the mitotic spindle, microtubules of the spindle physically compete for binding to the same site on the kinetochore where MPS1 is bound. Thus, once the kinetochore is properly attached, MPS1 dissociates, the inhibitory signal is lost, and cell division is allowed to proceed. Science, this issue pp. 1264 and 1260
\end{abstract}

ARTICLE TOOLS

SUPPLEMENTARY MATERIALS

RELATED

CONTENT

REFERENCES

PERMISSIONS http://science.sciencemag.org/content/348/6240/1264

http://science.sciencemag.org/content/suppl/2015/06/10/348.6240.1264.DC1

http://science.sciencemag.org/content/sci/348/6240/1260.full

This article cites 27 articles, 11 of which you can access for free http://science.sciencemag.org/content/348/6240/1264\#BIBL

http://www.sciencemag.org/help/reprints-and-permissions

Use of this article is subject to the Terms of Service

Science (print ISSN 0036-8075; online ISSN 1095-9203) is published by the American Association for the Advancement of Science, 1200 New York Avenue NW, Washington, DC 20005. The title Science is a registered trademark of AAAS.

Copyright $@ 2015$, American Association for the Advancement of Science 